\title{
Study of Turbulent Models in Jet Impingement at Different Cross-Flow Conditions
}

\author{
\{Abd Elnaby Kabeel, Medhat Elkelawy*, Hagar Alm-Eldin Bastawissi,
} Ahmed Mohammed El-Banna $\}^{\dagger}$

\begin{abstract}
Air impingement jet into main cross stream is countered in numerous modern applications, for example, dryers, pneumatic conveying and spot cooling. The common impact between the jet and the cross stream is analyzed at various jet to cross flow velocity ratio. In the present study, an air jet is impinged at an assortment of velocity ratios into a cross stream. The cross stream is brought out through a $10 \mathrm{~cm}$ diameter pipe with Reynolds number reaches out $6 \times 10^{4}$. At the diverse conditions, the flow pattern is simulated numerically with two-equation turbulent models of Realizable $\boldsymbol{k}-\boldsymbol{\varepsilon}$, SST $\boldsymbol{k}-\boldsymbol{\omega}$ and RSM. The outcome demonstrates that the jet pattern is altogether misshaped as the standard speed is expanded and detachment regions are created. Greater state of turbulent intensity and stresses is seen in the regular face between the stream and cross stream.
\end{abstract}

Keywords: Jet Cross-Flow, Turbulent Models, Impinging jets, Three Dimensions Computational Fluid Dynamics (3D-CFD)

\section{Nomenclature}

$\begin{array}{ll}J I C & \text { Jet into Cross-flow } \\ V R & \text { Velocity Ratio } \\ \mathbf{R e}_{\text {cross }} & \text { Cross flow Reynolds number } \\ \mathrm{Re}_{\mathrm{j}} & \text { Jet Reynolds number } \\ \mathrm{D} & \text { Cross flow pipe diameter } \\ d & \text { Jet pipe diameter } \\ U, V, W & \text { Mean velocity in the } \mathrm{x}, \mathrm{y} \text { and } \mathrm{z} \text { directions, respectively } \\ U_{c r o s s} & \text { Mean velocity component of the cross flow at the inlet } \\ V J & \text { Mean velocity component of the jet at the inlet } \\ \overline{u^{\prime} u^{\prime}}, \overline{v^{\prime} v^{\prime}}, \overline{w^{\prime} w^{\prime}} & \text { Normal components of the mean specific Reynolds stress tensor in the } \\ \overline{u^{\prime} v^{\prime}} & \mathrm{x}, \mathrm{y}, \text { and z directions, respectively. } \\ & \text { Shear component of the specific Reynolds stress tensor components in } \\ C V P & \text { the x and y directions } \\ I & \text { Counter-rotating Vortex Pair }\end{array}$

* Asst. Prof, medhatelkelawy@f-eng.tanta.edu.eg,

$\dagger \quad$ Mechanical Power Eng. Dep., Faculty of Eng., Tanta University, Tanta, Egypt. 


\section{Introduction}

The jet into a cross stream are experienced in a various designing and ecological applications. For instance, air injection into coal burner, air injection into pneumatic conveying, and the jet of dilution mixture after the gas turbine combustor in order to weaken and cool the high temperature products before they enter the turbine sector, and finally for other modern applications to improve the mixing procedure.

Simulation of turbulent streams mixing is of great interest. The expanding computational facilities permit the simulation of bigger more complicated frameworks in shorter time frames. The scattering of this innovation accompanies new difficulties, as the expanded concentrate on the robustness, accuracy and models predictability, which are encountered with more complicated frameworks. The flow field where the jet is discharged into the cross flow could be classified into three categories [1,2]; the potential core zone, the zone of maximum deflection, and the far field zone. The potential centre (core) zone is the focal part of the jet which extends from the jet source to few time of jet diameter. At the begging the developing jet interacts with cross-flow and generates shell-shape shear layer that is developed at jet boundaries. The shear layer surrounds the core so that it will not be more affected by the cross flow however its length is little when compared with the jet in stagnant environment. The second region, where the jet experiences extreme deflection as a result of the shearing applied by cross flow. This region is characterized by, turbulent mixing field around the jet and strong interaction with cross-flow. In the far field zone, the jet undergoes in cross-flow direction asymptotically and the jet velocity is close to the cross-flow velocity.

The interaction amongst jet and cross stream includes the arrangement of different turbulent vortices and complex flow structures. including the counter-rotating vortex pair (CVP), the horseshoe vortex, the wake vortex, and the leading-edge and lee-side shear layer vortices[3].The CVP is the primary element of JIC which has the major contribution to the enhanced mixing and still exists in the jet far-field.

As a result of its colossal applications and complex fluid stream phenomenon observed, the issue has been investigated experimentally and numerically by a few scientists amid the most recent decades. Investigations on the jet into cross flow (JIC) have started in 1970's. Margason[4] surveys various examinations of the JIC configuration, much of them pay particular attention to the complex framework of vortices and their impact to the flow field stability. Some modern references utilizing laser diagnostics on the JIC have been per-framed under weak levels of turbulence with low Reynolds numbers these conditions seem irrelevant to the widespread applications of JIC[5],[6]. Furthermore, experimental results were obtained for round jet in cross-flow using planer laser induced fluorescence[7]. The velocity field characteristics have captured for velocity ratio of 5.7 and jet exit Reynolds number of 5000. In another work the turbulent fluctuations for JIC were measured using Particles Image velocimetry (PIV)[8]. The mixing process was investigated at cross-flow Reynolds number of 3000 and $\mathrm{VR}=3$.

With the progression of innovation in both exploratory and numerical fields, numerous new characteristics of the stream field were resolved in the research, for example, the impacts of the jet to cross-flow speed ratio, injection inclination angle, jet and cross-flow Reynolds numbers. Numerous approaches have been used to characterize the JIC in a cross-flow, especially numerical modeling [9]. Using two RANS models namely k- $\varepsilon$ and SST in addition with the large eddy simulation (LES) turbulent models performed to characterize the mixing process for $\mathrm{VR}=4$.

A round stream ejected into a bound cross-flow in a rectangular passage has been investigated computationally using standard $\mathrm{k}-\varepsilon$ turbulence model and experimentally. The main result is 
that the turbulent Schmidt number significantly affects the expectation of the species spreading rate in jet in cross-flows, particularly for the situations where the jet to-cross-flow momentum flux proportions are generally little[10]. Computational and experimental investigation have performed for turbulent Jet into cross-flow is investigated at VR equal to 2 and 4 [11]. The impact of jet angle was examined by simulation techniques [12]. The standard $\mathrm{k}-\varepsilon$ and RANS models were used for a jet directed normal and oblique to cross-flow. The cross-flow mass ratio was varied from 0.005 to 1 with different jet inclination angles.

The current study aims to take the advantage of simulation and overcomes the limiting conditions which are associated with the experimental work. This is accomplished by simulating the three-dimensional turbulence flow field within JIC under highly turbulent conditions. The arrangement of various types of vortices and their impacts on the mean speed is examined. To investigate precision of the computational results, the simulation outcomes are compared with the experimental results of early work of [9] and a similar boundary conditions based on the dimensionless principles is guaranteed for both. In the present work, the numerical examinations were made utilizing three diverse turbulence models, to be specific, the Realizable k- $\varepsilon$, SST k- $\omega$ and RSM.

\section{Numerical Approach}

The transport governing equations for mass, momentum and the turbulent energy flow were solved using commercial CFD program ANSYS Fluent Release 16.0[13]. The package solves the equations using finite volume method. The domain for the present computational work was selected to use uniform velocity profile at the inlets with developing boundary layer inside the domain. As shown in Figure 1 the domain is composite of $\mathrm{L}=4000 \mathrm{~mm}$ long horizontal pipe with diameter of $\mathrm{D}=100 \mathrm{~mm}$ and perpendicular jet pipe directed downward to discharge the jet into the cross-flow vertically. The jet pipe diameter $\mathrm{d}=25.4 \mathrm{~mm}$ and length of $(\mathrm{L}>10 \mathrm{~d})$ to ensure a fully developed velocity profile at the jet outlet. The jet flow exit is placed $1750 \mathrm{~mm}$ downstream the beginning of the cross-flow pipe to guarantee a fully developed flow is present prior to the jet. The original of the coordinates system is located at the centerline of the pipe where the jet axis is intersected with it and the $\mathrm{x}$ axis in the stream wise direction of the cross flow and the $\mathrm{z}$ axis coincides with the jet axis.

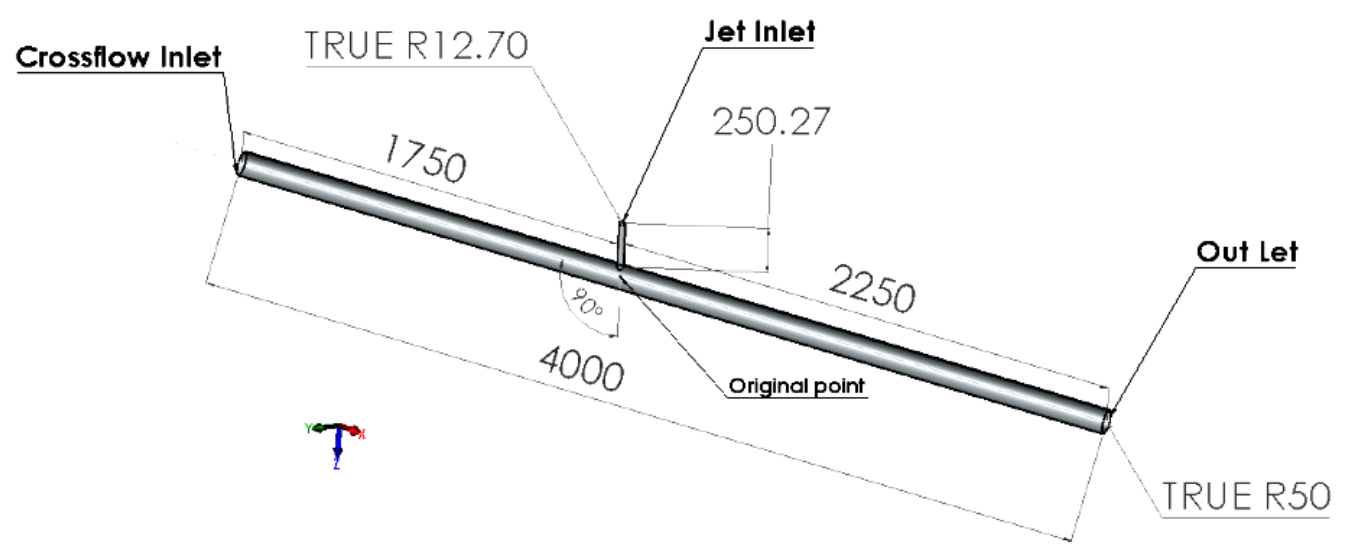

Fig. 1. Schematic illustration for Jet-Into-Cross-flow 


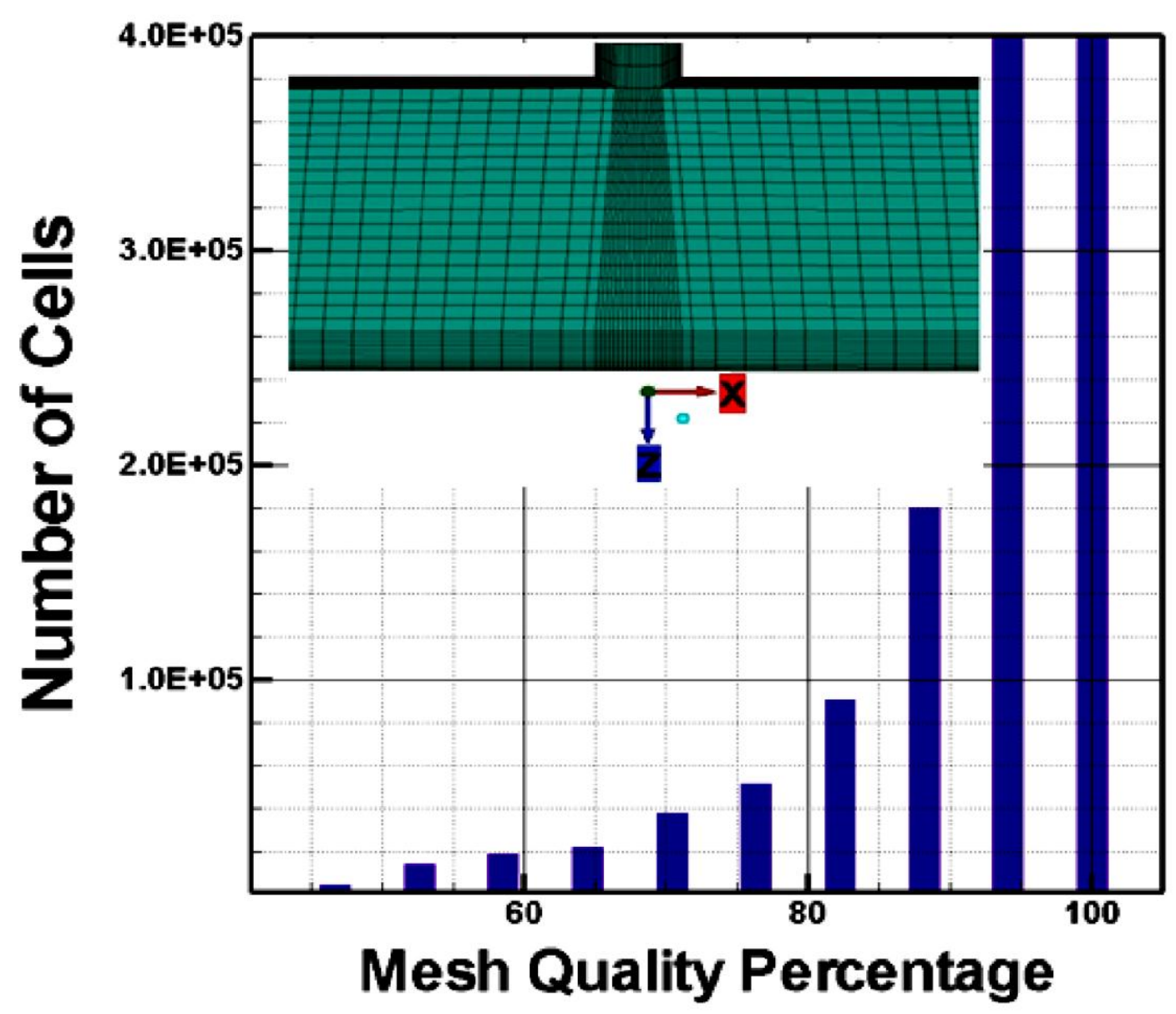

Fig. 2. Computational grid on the symmetry plane and histogram of the generated computational mesh

The computational grid was formed from 835316 cells divided to 792186 hexahedral shaped, 42826 quad shaped, and 304 line elements. The domain divided into two regions, first the near jet region that is characterized by finer grid. The second region is the far field region with relatively coarse grid. The domain for the present computational work and histogram of the mesh quality is illustrated in Figure 2. About ninety percent of the cell elements had a quality more than $80 \%$.

\subsection{Governing Equation}

The domain is governed by fully three dimensional transport equations for mass, momentum and energy. The flow turbulence modeling was based on the Reynolds-averaged equations in which decomposed the flow variables into time-averaged and fluctuating components[14]. The Reynolds-averaged Nervier-Stokes (RANS) in Cartesian form for incompressible flow are described as the following:

$$
\begin{aligned}
& \frac{\partial \rho}{\partial t}+\frac{\partial}{\partial x_{i}}\left(\rho U_{i}\right)=0 \\
& \frac{\partial}{\partial t}\left(\rho U_{i}\right)+\frac{\partial}{\partial x_{j}}\left(\rho U_{i} U_{j}\right)=-\frac{\partial P}{\partial x_{i}}+\frac{\partial}{\partial x_{j}}\left[\mu\left(\frac{\partial U_{i}}{\partial x_{j}}+\frac{\partial U_{j}}{\partial x_{i}}-\frac{2}{3} \delta_{i j} \frac{\partial U_{l}}{\partial x_{l}}\right)\right]+\frac{\partial}{\partial x_{j}}\left(-\rho \overline{u_{i}^{\prime} u_{j}^{\prime}}\right)
\end{aligned}
$$


The solution variables $\mathrm{P}$ and $\mathrm{Ui}$ are the ensemble averaged pressure and velocity components respectively. Where ${ }^{\prime}{ }_{i}^{\prime}$ are the fluctuating velocity components, ${ }^{x_{i}}$ is the coordinate direction. However additional unknown terms called Reynolds stresses $\left(-\rho \overline{u_{i}^{\prime} u_{j}^{\prime}}\right)$, originate from the averaging process as a result of turbulent motion. Their solution requires turbulence modelling in order to provide an appropriate closure for the sets of equations.

\subsection{Turbulence Modeling}

The eddy viscosity model is the closure based on Boussinesq hypothesis relation relates the Reynolds stresses and the mean strain rate.

$$
\tau_{i j}=-\rho \overline{u_{i}^{\prime} u_{j}^{\prime}}=\mu_{t}\left(\frac{\partial U_{i}}{\partial x_{j}}+\frac{\partial U_{j}}{\partial x_{i}}\right)-\frac{2}{3}\left(\rho k+\mu_{t} \frac{\partial U_{k}}{\partial x_{k}}\right) \delta_{i j}
$$

The Boussinesq approach characterized by lower computational effort beside the turbulent eddy viscosity $\mu_{\mathrm{t}}$ calculated using empirical formula as a function of the kinetic energy of turbulence and the turbulent length scale. Two additional transport equations are required to model the eddy viscosity one equation for the turbulent kinetic energy $\mathrm{k}$, and other one for either the turbulence dissipation rate $\varepsilon$ or the specific dissipation rate $\boldsymbol{\omega}$. The details of their formulation are described below.

\subsubsection{Realizable $k-\varepsilon$ Model}

The Realizable $\boldsymbol{k}-\boldsymbol{\varepsilon}$ turbulence model attempts to satisfy certain mathematical constraints on the Reynolds stresses, which they are in agreement with the physics of turbulent flows[15]. The Realizable $\boldsymbol{k}-\boldsymbol{\varepsilon}$ is characterized by a modified model equation for dissipation $(\varepsilon)$ based on the dynamic equation of the mean-square vorticity. The turbulent kinetic energy $\boldsymbol{k}$ and the turbulence dissipation rate $\varepsilon$ are governed by the following transport equations.

$$
\begin{aligned}
& \frac{\partial}{\partial t}(\rho k)+\frac{\partial}{\partial x_{j}}\left(\rho k U_{j}\right)=\frac{\partial}{\partial x_{j}}\left[\left(\mu+\frac{\mu_{t}}{\sigma_{k}}\right) \frac{\partial k}{\partial x_{j}}\right]+G_{k}+G_{b}-\rho \varepsilon-Y_{M} \\
& \frac{\partial}{\partial t}(\rho \varepsilon)+\frac{\partial}{\partial x_{j}}\left(\rho \varepsilon U_{j}\right)=\frac{\partial}{\partial x_{j}}\left[\left(\mu+\frac{\mu_{t}}{\sigma_{\varepsilon}}\right) \frac{\partial \varepsilon}{\partial x_{j}}\right]+\rho C_{1} S \varepsilon-\rho C_{2} \frac{\varepsilon^{2}}{k+\sqrt{v \varepsilon}}+C_{1 \varepsilon} \frac{\varepsilon}{k} C_{3 \varepsilon} G_{b}
\end{aligned}
$$

Where $G_{k}$ is the turbulent production term represents the turbulent kinetic energy generation due to mean velocity gradient. This term is defined as

$$
G_{k}=-\rho \overline{u_{i}^{\prime} u_{j}^{\prime}} \frac{\partial U_{i}}{\partial x_{j}}
$$

$\mathrm{G}_{\mathrm{b}}$ is the turbulent kinetic energy generation due to bouncy. $\mathrm{Y}_{\mathrm{M}}$ is the contribution of the fluctuating dilatation in compressible turbulence to the overall dissipation rate. The quantities $\sigma_{\mathrm{k}}$ and $\sigma_{\varepsilon}$ are the turbulent Prandtl numbers for $\boldsymbol{k}$ and $\boldsymbol{\varepsilon}$, they are equal to 1 and 1.2 respectively. $\mathrm{C}_{2}$ and $\mathrm{C}_{1 \varepsilon}$ are constants and equal to 1.9 and 1.44 , respectively. The turbulent viscosity $\mu_{t}$ is written as the following;

$$
\mu_{t}=\rho C_{\mu} \frac{k^{2}}{\varepsilon}
$$


The Realizable $\boldsymbol{k}-\boldsymbol{\varepsilon}$ has proposed alternative formulation for the turbulent viscosity by involving a variable $C_{\mu}$. For the large strained mean flows the rule of positive normal Reynolds stress may be violated which is non-realizable. The most reasonable approach to guarantee a realizable normal Reynolds stress is to make $C_{\mu}$ variable. It is written as

$$
C_{\mu}=\frac{\mathrm{I}}{A_{\circ}+A_{s} \frac{k U^{*}}{\varepsilon}}
$$

Where $\mathrm{A}_{\mathrm{o}}=4.04, \mathrm{~A}_{\mathrm{s}}=\sqrt{6} \cos \phi \cos \phi, U^{*} \equiv \sqrt{S_{i j} S_{i j}+\tilde{\Omega}_{i j} \tilde{\Omega}_{i j}} \quad, \tilde{\Omega}_{i j}=\Omega_{i j}, \Omega_{i j}=\bar{\Omega}_{i j}-\varepsilon_{i j k} \omega_{k}$ and $\bar{\Omega}_{i j}$ is the main rate of rotation tensor.

\subsubsection{Shear-Stress Transport (SST) k- $\omega$}

This section exhibits the Shear-Stress Transport (SST) model results from a modification to the definition of the eddy-viscosity in the Baseline (BSL) k- $\omega$ Model, which takes into consideration the impact of the transport of the principal turbulent shear stress [16]. The modified model introduces improvements in the prediction of adverse pressure gradient flows. The major differences amongst SST and the standard $\mathbf{k}-\boldsymbol{\omega}$ model are the gradual change from the standard k- $\omega$ model in the inner region of the boundary layer to a high-Reynolds number version of the k- $\varepsilon$ model in the outer part of the boundary layer. Also, modified turbulent viscosity formulation is introduced to account for the transport effects of the principal turbulent shear stress.

One of the powerless aspects of the standard k- $\omega$ model is the strong sensitivity of the solutions to values for $\mathrm{k}$ and $\omega$ outside the shear layer (free-stream sensitivity). The $\boldsymbol{k}-\boldsymbol{\varepsilon}$ model is converted into k- $\omega$ model and employed in the far field region while the standard $\boldsymbol{k}-\boldsymbol{\omega}$ model is activated near the wall region with no dependence on free-stream. The standard $\boldsymbol{k}-\boldsymbol{\omega}$ model and the transformed k- $\boldsymbol{\varepsilon}$ model are both multiplied by a blending function and both models are added together. The blending function is designed to be one in the near-wall region, which activates the standard k- $\omega$ model, and zero away from the surface, which activates the transformed $\mathbf{k}-\boldsymbol{\varepsilon}$ model. The SST $\mathbf{k}-\boldsymbol{\omega}$ model is governed by the transport equations as the following;

$$
\begin{aligned}
& \frac{\partial}{\partial t}(\rho k)+\frac{\partial}{\partial x_{j}}\left(\rho k U_{j}\right)=\frac{\partial}{\partial x_{j}}\left(\Gamma_{k} \frac{\partial k}{\partial x_{j}}\right)+G_{k}-Y_{k} \\
& \frac{\partial}{\partial t}(\rho \omega)+\frac{\partial}{\partial x_{j}}\left(\rho \omega U_{j}\right)=\frac{\partial}{\partial x_{j}}\left(\Gamma_{\omega} \frac{\partial \omega}{\partial x_{j}}\right)+G_{\omega}-Y_{\omega}+D_{\omega}
\end{aligned}
$$

Where $G_{k}$ is the turbulent production term represents the turbulent kinetic energy generation due to mean velocity gradient, defined as in the realizable $k-\varepsilon$ section. $G_{\omega}$ is the generation of $\omega$ and defined as:

$$
G_{\omega}=\frac{\alpha \alpha^{*}}{v_{t}} G_{k}
$$

Where $v_{t} \equiv k / \omega$ and $\alpha^{*}$ is the turbulent viscosity damping coefficient which represents a correction factor for low Reynolds number, described as: 
$\alpha^{*}=\alpha_{\infty}^{*}\left(\frac{\alpha_{0}^{*}+\operatorname{Re}_{t} / \mathrm{R}_{k}}{1+\operatorname{Re}_{t} / \mathrm{R}_{k}}\right)$

Where, $\operatorname{Re}_{t}=\frac{\rho k}{\mu \omega}, \mathrm{R}_{\mathrm{k}}=6, \alpha_{0}^{*}=\frac{\beta_{i}}{3}$ and $\beta_{i}=0.072$.

The major feature of the SST $\boldsymbol{k}$ - $\boldsymbol{\omega}$ model is the ability to take into consideration the transport of the turbulence shear stress implicitly in the turbulent viscosity definition as follow.

$\mu_{t}=\frac{\rho k}{\omega} \frac{1}{\max \left[\frac{1}{\alpha^{*}} \frac{S F_{2}}{\alpha_{1} \omega}\right]}$

The viscous heating effect could be included in the same manner as for the Realizable k- $\varepsilon$ model. The drawback of two equation models is the over predicting of turbulence generation near the stagnation regions. This behavior is avoided by activating the turbulence production limiter which limits the growth of the production term $\mathrm{G}_{\mathrm{k}}$, defined as follow

$$
G_{k}=\min \left(G_{k}, C_{\text {lim }} \rho \varepsilon\right)
$$

This modification is employed automatically for $\boldsymbol{k}$ - $\boldsymbol{\omega}$ model. Within ANSYS fluent, the SST model uses the automatic near-wall treatment, which allows for a smooth shift from a lowReynolds number to a wall-function formulation

\subsubsection{Reynolds Stress Model (RSM)}

The Reynolds Stress Model [17, 18]is an alternative method for closing the Reynolds averaged Navier Stokes equations where the Reynolds stress and fluxes are modeled. The RSM aims to solve transport equations of each term of Reynolds stresses tensor and additional equation for eis required. The transport Reynolds stress equation is derived by taking the moments of the momentum equation. This is done by multiplying the exact momentum equations for the fluctuations by the fluctuating velocities and averaged. The transport Reynolds stress equation describes as the following equations;

$$
\begin{aligned}
& \left.\frac{D \tau_{i j}}{D t}=\frac{\partial}{\partial t}\left(\rho \overline{u_{i}^{\prime} u_{j}^{\prime}}\right)+\frac{\partial}{\partial x_{k}}\left(\rho U_{k} \overline{u_{i}^{\prime} u_{j}^{\prime}}\right)=-\frac{\partial}{\partial x_{k}}\left(\rho \overline{u_{i}^{\prime} u_{j}^{\prime} u_{k}^{\prime}}+\overline{p^{\prime}\left(\delta_{k j} u_{i}^{\prime}+\delta_{i k} u_{j}^{\prime}\right.}\right)\right) \\
& +\frac{\partial}{\partial x_{k}}\left(\mu \frac{\partial}{\partial x_{k}}\left(\overline{u_{i}^{\prime} u_{j}^{\prime}}\right)\right)-\rho\left(\overline{u_{i}^{\prime} u_{k}^{\prime}} \frac{\partial U_{j}}{\partial x_{k}}+\overline{u_{i}^{\prime} u_{k}^{\prime}} \frac{U_{i}}{\partial x_{k}}\right)+\overline{p^{\prime}\left(\frac{\partial u_{i}^{\prime}}{\partial x_{j}}+\frac{\partial u_{j}^{\prime}}{\partial x_{i}}\right)}-2 \mu \frac{\partial u_{i}^{\prime}}{\partial x_{k}} \frac{\partial u_{j}^{\prime}}{\partial x_{k}}
\end{aligned}
$$

where

$$
\begin{aligned}
& C_{i j}=\frac{\partial}{\partial x_{k}}\left(\rho U_{k} \overline{u_{i}^{\prime} u_{j}^{\prime}}\right) \\
& D_{T, i j}=-\frac{\partial}{\partial x_{k}}\left(\rho \overline{u_{i}^{\prime} u_{j}^{\prime} u_{k}^{\prime}}+\overline{p^{\prime}\left(\delta_{k j} u_{i}^{\prime}+\delta_{i k} u_{j}^{\prime}\right)}\right) \\
& D_{L, i j}=\frac{\partial}{\partial x_{k}}\left(\mu \frac{\partial}{\partial x_{k}}\left(\overline{u_{i}^{\prime} u_{j}^{\prime}}\right)\right)
\end{aligned}
$$




$$
\begin{aligned}
P_{i j} & =-\rho\left(\overline{u_{i}^{\prime} u_{k}^{\prime}} \frac{\partial U_{j}}{\partial x_{k}}+\overline{u_{i}^{\prime} u_{k}^{\prime}} \frac{U_{i}}{\partial x_{k}}\right) \\
\phi_{i j} & =p^{\prime}\left(\frac{\partial u_{i}^{\prime}}{\partial x_{j}}+\frac{\partial u_{j}^{\prime}}{\partial x_{i}}\right) \\
\varepsilon_{i j} & =-2 \mu \frac{\partial u_{i}^{\prime}}{\partial x_{k}} \frac{\partial u_{j}^{\prime}}{\partial x_{k}}
\end{aligned}
$$

where $C_{i j}, D_{T, i j}, D_{L, i j}, P_{i j}, \phi_{i j}$ and $\varepsilon_{i j}$ are the convection term, turbulent diffusion, molecular diffusion, stress production, pressure strain and dissipation, respectively. The terms $D_{T, i j}$, $\phi_{i j}$ and $\varepsilon_{i j}$ must be modeled in order to close the equations. The turbulent diffusion term is modeled using generalized gradient diffusion model[19], and the pressure strain term has modeled using quadratic pressure strain model[20].

\subsection{Boundary Conditions}

As opposed to other papers which utilize for the most part weak turbulent conditions to examine the JIC, the present investigations were performed under exceptionally high turbulent conditions. The flow boundary conditions are illustrated in Table1. It is a common practice to characterize JIC using the velocity ratio, VR, which is defined as the square root of the momentum ratio[21] which are described as follow;

$$
V R=\sqrt{\frac{\rho_{j} W_{j}^{2}}{\rho_{\text {cross }} U_{\text {cross }}^{2}}}
$$

Where $\mathrm{W}_{\mathrm{j}}$ is the mean jet velocity component in $\mathrm{z}$ direction and $\mathrm{U}$ cross is the mean cross-flow velocity in $\mathrm{x}$ direction. In the case of equal densities for the cross flow and the jet the VR can be written as $\mathrm{VR}=\mathrm{W}_{\mathrm{j}} / \mathrm{U}$ cross. The mean velocity at the inlets was chosen as characteristic velocity. For the current flow conditions, the velocity ratio takes the value 2.776 . However, a no-slip boundary condition was applied at the walls. The boundary condition for the turbulence intensity (I) has obtained using the following formula

$$
I \equiv \frac{u^{\prime}}{U}=0.16(\mathrm{Re})^{-1 / 8}
$$

Table 1. Boundary Conditions

\begin{tabular}{|c|c|}
\hline Cross flow inlet & Right \\
\hline $\mathrm{U}_{\text {cross }}=14 \mathrm{~m} / \mathrm{s}$ & $\mathrm{W}_{\mathrm{j}}=38.864 \mathrm{~m} / \mathrm{s}$ \\
$\mathrm{T}_{\text {cross }}=25 \mathrm{C}^{\mathrm{o}}$ & $\mathrm{T}_{\mathrm{j}}=25 \mathrm{C}^{\mathrm{o}}$ \\
$v_{\text {cross }}=1.5571 \times 10^{-5} \mathrm{~m}^{2} / \mathrm{s}$ & $v_{\mathrm{j}}=1.5571 \times 10^{-5} \mathrm{~m}^{2} / \mathrm{s}$ \\
$\mathrm{Re}_{\text {cross }}=\mathrm{U}_{\text {cross }} \mathrm{D} / \mathrm{v}_{\text {cross }}=$ & $\mathrm{Re}_{\mathrm{jet}}=\mathrm{U}_{\text {cross }} \mathrm{D} / \mathrm{v}_{\text {cross }}=$ \\
89911 & 63396.4 \\
$\mathrm{I}=5 \% \quad \mathrm{I}=5 \%$ \\
\hline \multicolumn{2}{|c|}{$\mathrm{VR}=2.776$} \\
\hline
\end{tabular}




\subsection{Numerical Algorithms}

The transport governing equations for mass, momentum and energy governing the turbulent flow were solved using commercial CFD program ANSYS Fluent Release 16.0[13]. The package solves the equations using finite volume method in which the governing equations are integrated over each control volume to construct algebraic discretized equations. The discretized equations are then linearized to form linear equation system. The linear system is solved using implicit Gauss-Seidel linear equation solver.

The transport equations are solved using pressure-based solver where the velocity field is obtained from the momentum equation. However, the pressure field is obtained from the pressure correction equation which is obtained by manipulating continuity and momentum equation. In this method the velocity field is corrected by the pressure to satisfy the continuity. Therefore the governing equations are nonlinear and coupled to each other which require that iterative procedures have accomplished through the entire set of the governing equations. The momentum and pressure-based continuity equations are solve simultaneously using fully implicit coupling algorithm.

The diffusion terms are discretized using central deference second order scheme. However, the quantities of variable at cell faces are required for the convection terms; therefore, an interpolation scheme is used to compute the face values from the cell values. The second order interpolation scheme is used for the pressure term. The second-Order upwind scheme interpolates the face values which are required for the momentum equation from the cell centre values. The gradients are computed using Least squares Cell-based method. The linear system is solved using iterative techniques, the underrelaxation factors were 0.3 for momentum and pressure, and equal 0.5 for both turbulent kinetic energy and turbulent dissipation rate. The solution convergence is achieved as the normalized residual for the energy equals $10^{-6}$ and for the other variables equal $10^{-3}$.

\section{Results and Discussion}

\subsection{Degree Injection at Single Speed Ratio (VR)}

For the validation purpose a comparison has made with experimental and simulation results [9]. The validation based on non-dimensional similarity. The validation has conducted under similar boundary conditions based on the dimensionless principles. The direct comparison between the measured and simulated velocity was not possible without dimensionless principles. This is due to the geometrical and dimensional differences between the experimental and the simulation work. In order to eliminate the effects of the geometrical and dimensional effects, a customized form of Reynolds number is used and defined by the following equation.

$$
\operatorname{Re}^{*} x_{i}=\frac{U_{i} L}{v}
$$

Where $\mathrm{U}_{\mathrm{i}}$ is the characteristic velocity component, $\mathrm{L}$ is the characteristic length corresponding to the plane at which the velocity components are examined and $v$ is the kinematic viscosity. This number is found to be indicative of the corresponding velocity component. However, In the case of the measurements the characteristic length is chosen to equal $0.046 \mathrm{~m}$ while for the simulation equals to $0.0373 \mathrm{~m}$. This represents the distance between the cross flow centerline and the plane at which the velocity components are examined. Figure 3 shows the comparison between the measured and simulated $\mathrm{Re}^{*}$. In the case of measurements profiles are taken at $1 \mathrm{~d}$ above the jet inlet while for the simulation $0.5 \mathrm{~d}$ below the jet inlet. The 
simulations data represent both two-equation turbulence models and the RSM. The simulated velocity profiles in the $\mathrm{x}$ and $\mathrm{z}$ directions show good agreement with the measurements.

Figure 4 shows two dimensional plots of the PIV measurements at a plane located at $0.5 \mathrm{~d}$ below the jet inlet compared with the simulations data using the Realizable $\boldsymbol{k}-\boldsymbol{\varepsilon}$, SST $\boldsymbol{k}-\boldsymbol{\omega}$, and RSM turbulence models at a plane located at $0.5 \mathrm{~d}$ below the jet inlet for the velocity components $\mathrm{U}$ and $\mathrm{V}$ and the specific Reynolds stress component $\overline{u^{\prime} v^{\prime}}$. The geometrical differences between the measurements and the simulation are eliminated by normalized the dimensions of both.

The velocity fields are well represented by the three turbulence models with a small advantage to the k- $\varepsilon$ and SST models. The experimental specific Reynolds stress component $\overline{u^{\prime} v^{\prime}}$ shows a distinct character as two reversed peak values can be observed on the sides of the jet. The results from the simulations show a different behavior. However, the pattern is not in good agreement between the measurements and the simulations. In addition, the experimental values are higher and extended to wider region than the predictions from the simulations. Consequently, there is a more intense mixing at the lee side of the jet in the measurements than in the simulations.

The simulations data obtained by using the Realizable $\boldsymbol{k}-\boldsymbol{\varepsilon}$ turbulence model closes to the maxima and minima of the experimental data reasonably well. However, the distribution is incongruous. The simulation using the SST k- $\omega$ turbulence model predicts lower levels of Reynolds flux component. The Realizable $\boldsymbol{k}-\boldsymbol{\varepsilon}$ retains the upper hand by predicting higher turbulence levels than the SST $\boldsymbol{k}$ - $\boldsymbol{\omega}$ model and the RSM. Even though their predicted levels remain lower than in the measurements. And the spatial distribution of the Realizable $\boldsymbol{k}-\boldsymbol{\varepsilon}$ and RSM are very close to each other.

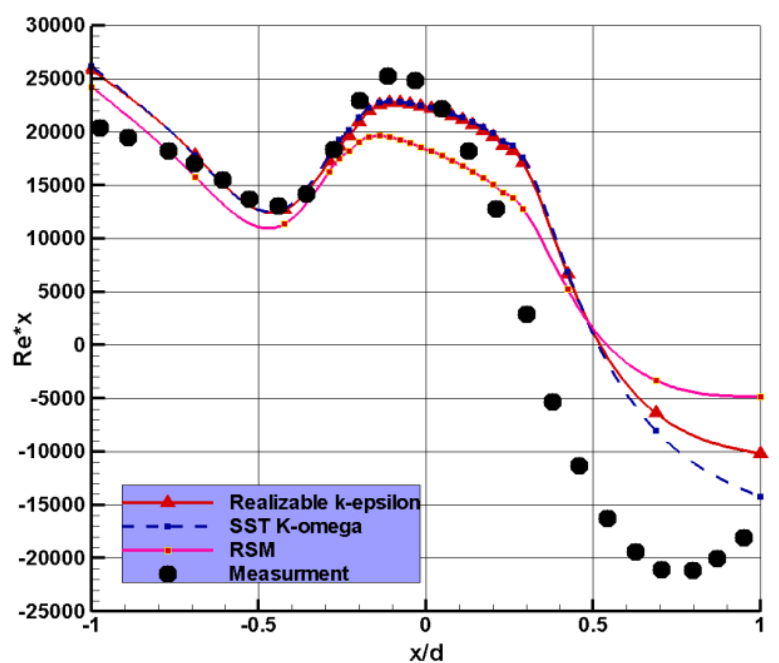

(a)

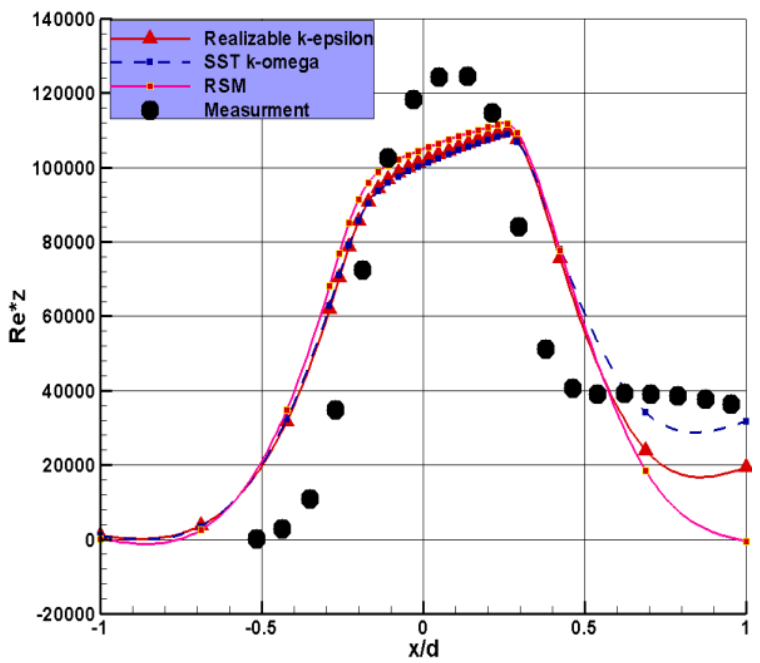

(b)

Fig. 3. Stream wise distribution of the (a) $\operatorname{Re}^{*} x$ and (b) $\operatorname{Re}^{*} \mathrm{z}$ at a plane $0.5 d$ below the jet inlet 


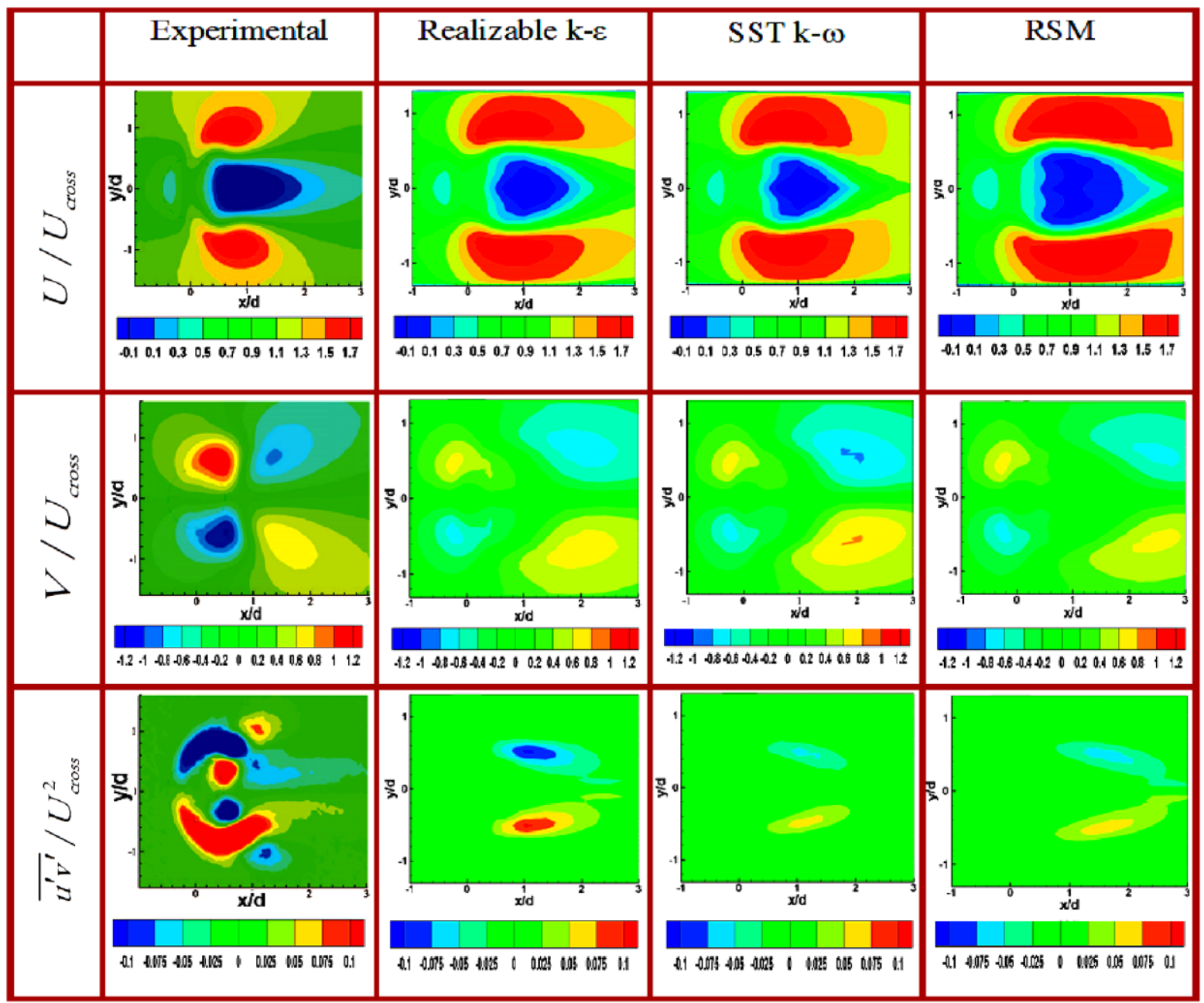

Fig. 4. Two dimensional contours at a plane located $0.5 \mathrm{~d}$ below the jet inlet

In Figure 5 the two-dimensional maps of the simulated Reynolds-stresses in $\mathrm{x}$ and $\mathrm{y}$ directions are depicted using the three models. The simulated specific Reynolds stress components $\overline{u^{\prime} u^{\prime}}$ and $\overline{V^{\prime} v^{\prime}}$ from RSM show a distinct character as two peak values can be observed on the sides of the jet; in the case of the $\overline{u^{\prime} u^{\prime}}$ component, directly on the jet downstream side, and in the case of the $\overline{v^{\prime} v^{\prime}}$ component, side and downstream of the jet. However, the results from the two-equation models show a different behavior. The $\overline{u^{\prime} u^{\prime}}$ component has peak values directly downstream closer to the jet inlet while the $\overline{v^{\prime} v^{\prime}}$ component shows only one peak region at the downstream portion of the jet. In addition, as in the case of $\overline{u^{\prime} v^{\prime}}$ as shown in Figure 4 the RSM predicts higher stresses components levels for $\overline{u^{\prime} u^{\prime}}$ and $\overline{V^{\prime} V^{\prime}}$ than that for SST $\boldsymbol{k}-\boldsymbol{\omega}$ and $\boldsymbol{k}-\boldsymbol{\varepsilon}$ models. Therefore the slower turbulence mixing rate is predicted for these models.

It can be seen that the specific Reynolds stress components are consistent with the measurements and simulated $\mathrm{Re}^{*}$. As shown in Figure 6, the peaks from the specific Reynolds stress components correspond to the regions of large mean velocity gradients for the two directions considered. For example the position of the maxima of $\overline{u^{\prime} u^{\prime}}$ and $\overline{W^{\prime} W^{\prime}}$ profile corresponds approximately to the position where the velocity gradients $\partial U / \partial X$ and $\partial W / \partial Z$ are maximum. 


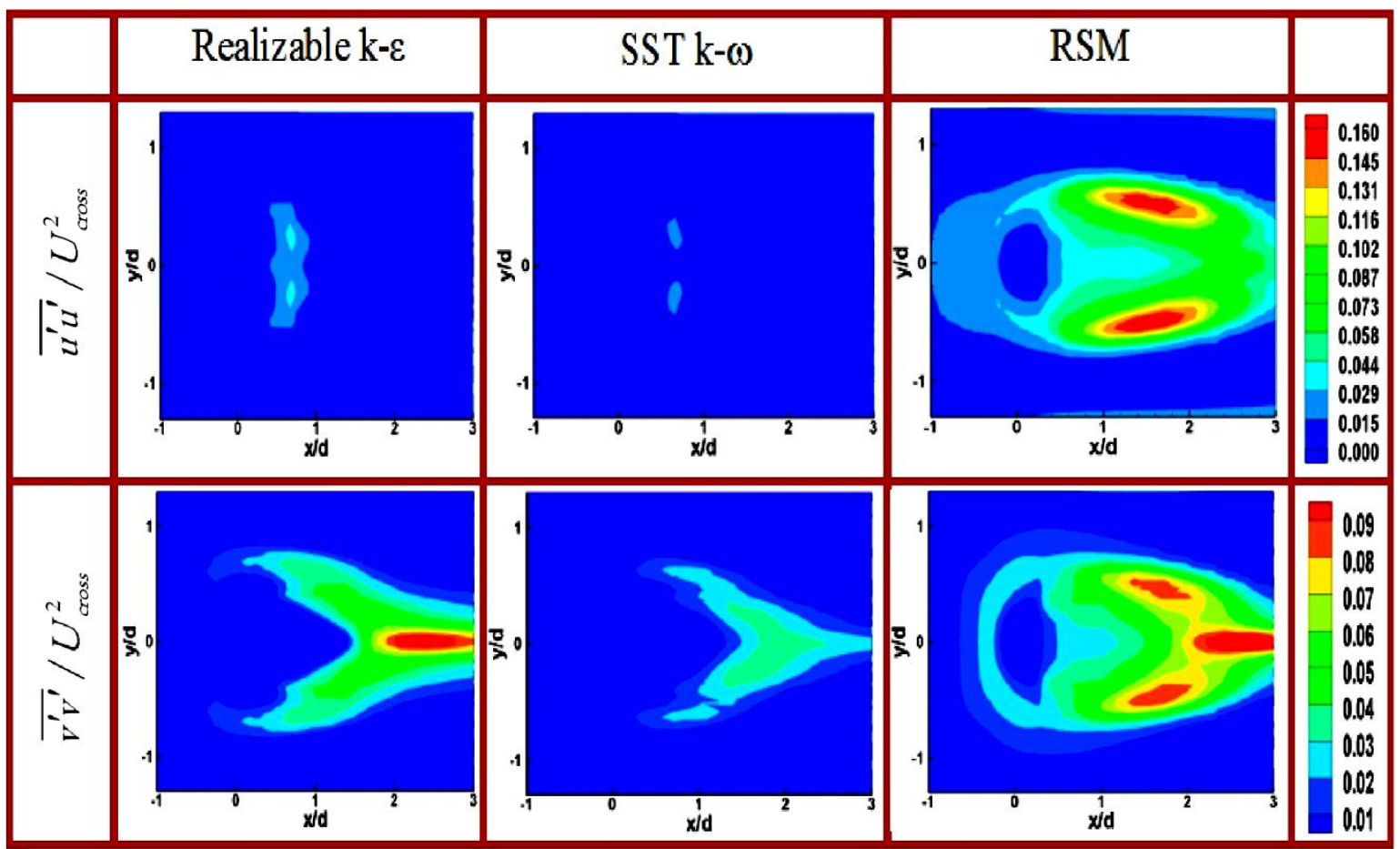

Fig. 5. Two dimensional contours at a plane located $0.5 \mathrm{~d}$ below the jet inlet

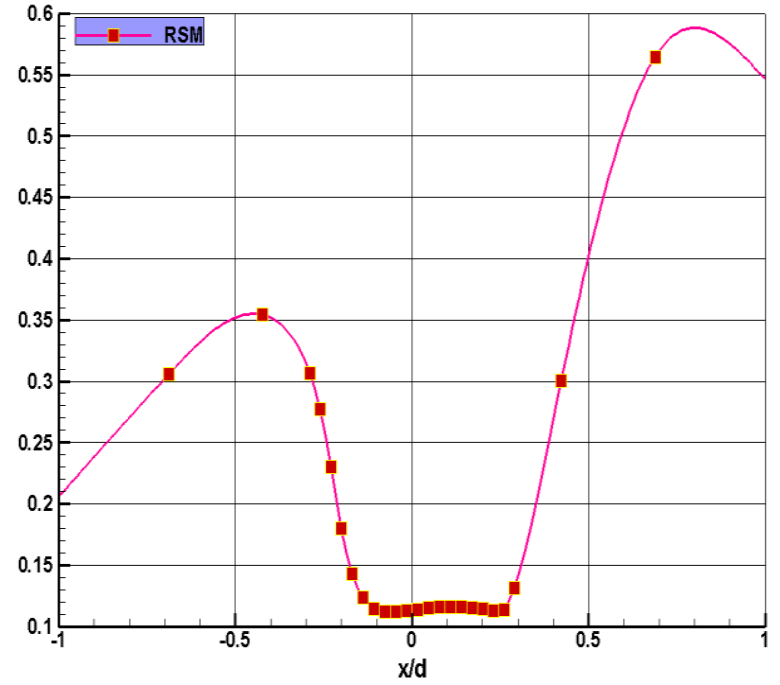

(a)

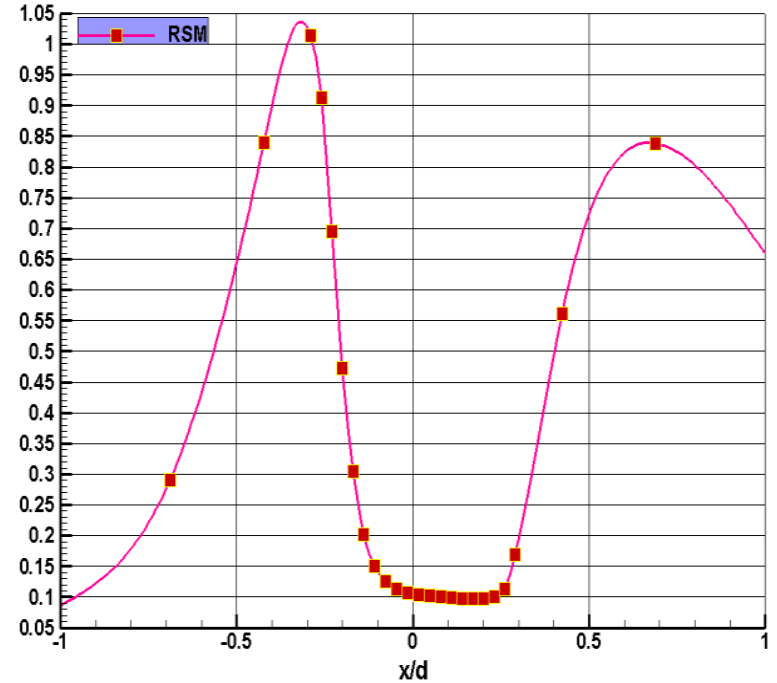

(b)

Fig. 6. Stream wise distribution of the Normal components of the specific Reynolds stress tensor (a) $\overline{u^{\prime} u^{\prime}}$ and (b) $\overline{W^{\prime} W^{\prime}}$ at a plane $0.5 \mathrm{~d}$ below the jet inlet obtained by RSM

One of the most important parameters of the JIC to engineering applications is the jet penetration, which is defined in this work as the locus of maximum U velocity. Figure 7 shows the comparison of the $U$ velocity component between values from both of twoequation models and RSM at the symmetry plane $\mathrm{y} / \mathrm{D}=0$. The maxima of axial velocity is higher for the RSM and excessive penetration predicting is observed in other word a longer distance corresponding to the locus of the maximum velocity. While for the two-equation models the axial velocity is relatively lower and the locus of the maximum axial velocity are is closer to the jet inlet. 
It can be seen that the jet penetration is very well represented by the simulations. Note that, the velocity profile prior to the jet is distorted by the presence of the horseshoe vortices and the profile has been prevented from being fully developed. Moreover, results show that when the two streams merge at the jet exit, first, the profile of the pipe flow becomes asymmetric, and second, the profile of the wall boundary layer is modified. Apparently, the cross-flow and the jet merge, with the cross-flow trying to slide past the jet. Consider the behavior of the stream wise velocity profile along the symmetry plane.

In the region close to the wall, we observe an initial deceleration of the cross-flow. Right downstream the jet exit; we observe a region with negative stream wise velocity corresponding to the upper part of the jet and confirming Figure 7 at $\mathrm{x} / \mathrm{d}$ equals to 1 and 2 . The recirculation region increases progressively as one move downstream of the jet exit. The recirculation regions caused by the combined actions of the wake vortices and the pair of CVP. This reverse flow acts to support the jet on the leeward side by inducing local upward lifting force to lift-off the jet from the wall. This translates into the rate of deceleration of the cross-flow as it travels around the jet as well as the magnitude of the adverse pressure gradient developed. Moving away from the wall $(\mathrm{y}=0)$, we see that the global flow tends to join the cross-flow velocity.

This downstream recirculation zone shows the presence of the phenomenon which is known as horseshoe tourbillion as shown in Figure 8. Horseshoe vortices, which form in the plane of the injection wall and surround the upstream portion of the jet, result from the interaction between the laminar (wall) boundary layer and the round transverse jet. The origin of these vortices is in the separation of the nozzle internal boundary layer from the leading edge wall. Horseshoe vortex system is found to be steady and oscillating. The horseshoe vortices are the first to take place near the injection plane just windward of the jet to finally wrap around the jet columns like a necklace. When the boundary layer's main flow fluid is deflected laterally from the center plane due to the adverse pressure gradient in front of the jet the span-wise vorticity in the boundary layer is stretched to form the front of the horseshoe vortices. Indeed, the streamlines just upstream the jet point will suffer a slight inclination toward the walls and will be deflected and entrained by the cross-flow. The vortices are shed along the front surface of the jet. The curvature of the leading-edge wall of the nozzle plays a key role in determining the shape of the vortices formed. The trajectories of these streamlines are definitely parallel to the upper boundary of the jet.

From Figure 9 and it becomes obvious that the fluctuations in the $\mathrm{x}$-direction exceed the corresponding quantity in the y-direction. It is evident that the interaction between the jet and the cross-flow is driven by the jet and the axial velocity component of the cross flow. The jet represents an obstacle for the cross flow on which the latter impinges. Therefore, the highest degree of fluctuation is expected for $\overline{u^{\prime} u^{\prime}} / U_{\text {cross }}^{2}$ in the vicinity of the center of the jet trajectory. The contour of $\overline{u^{\prime} u^{\prime}}$ maximum corresponds to the edges of the jet where $\partial U / \partial X$ is maximum. In these zones, where the highest fluctuations are observed, an intense mixing between the jet and the cross-flow is also taking place.

A significant velocity-component in the y-direction appears, when the cross-flow circulates around the jet which can be regarded as an obstacle. Therefore, it is evident that significant fluctuations of the flow in the y-direction $\overline{V^{\prime} V^{\prime}} / U_{\text {cross }}^{2}$ can only appear in the shear layer of the jet and the cross-flow where $\partial V / \partial Y$ is maximum. As a consequence, especially at heights just below the jet exit, zones of high fluctuations appear in a shell-like structure on the downstream side of the jet. At Lower highest the zones of high fluctuations are elongated as the jet is twisted by the cross flow and the y-direction velocity gradient is decreasing. Consequently, the maximum value of $\overline{V^{\prime} V^{\prime}}$ is decreasing. 

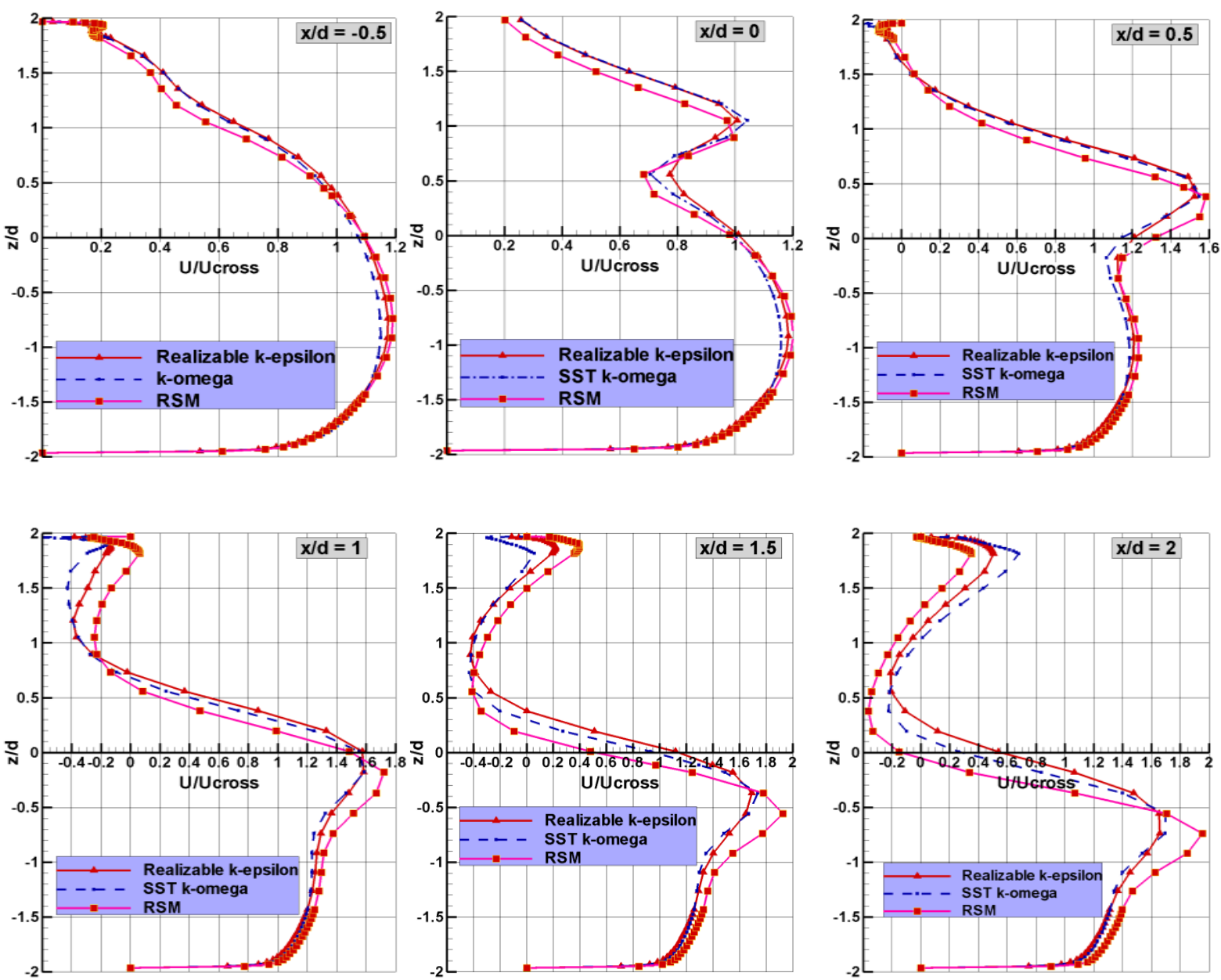

Fig. 7. Span wise distribution of the Normalized axial velocity component at the symmetry plane, $y / D=0$.

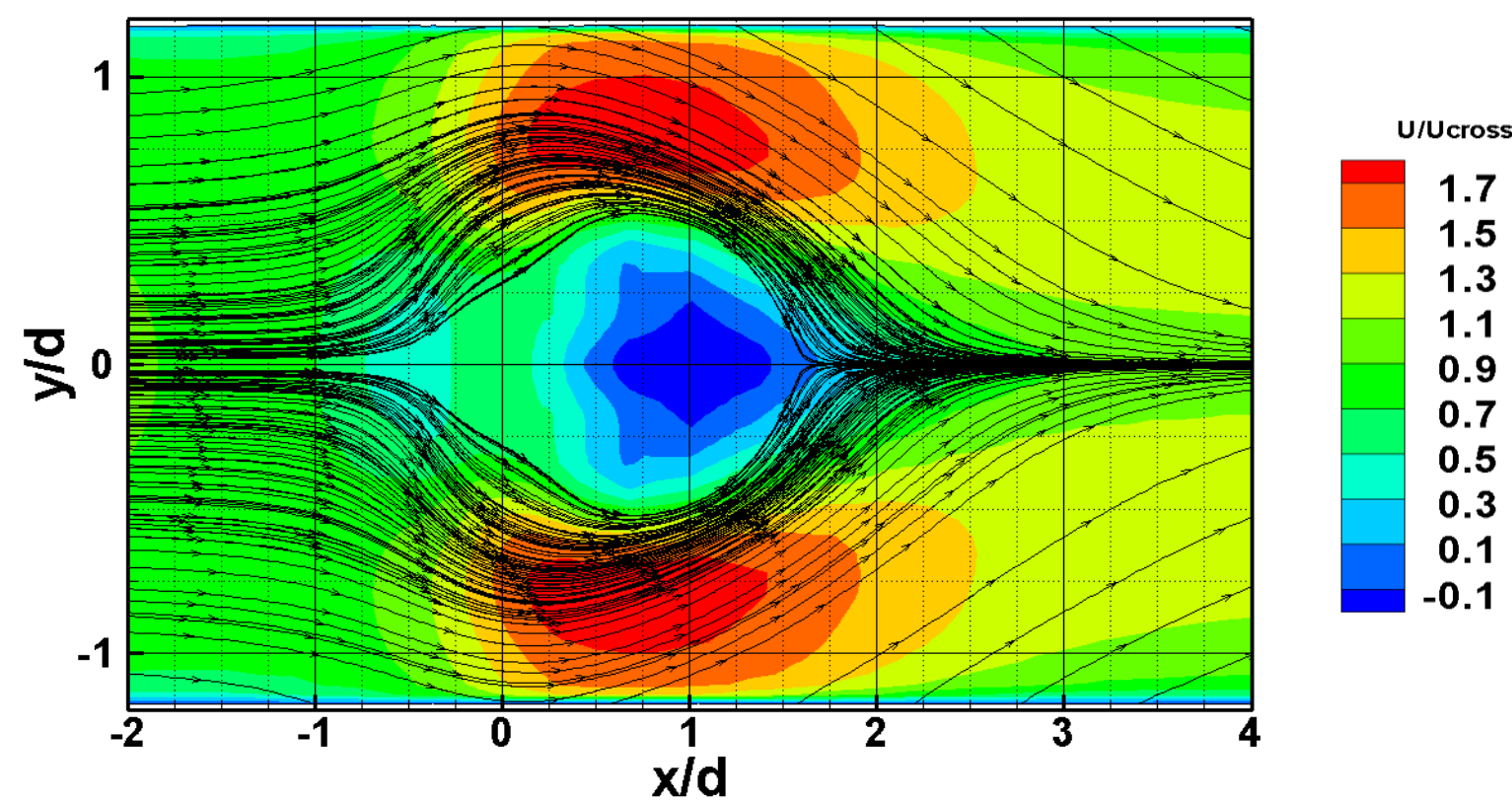

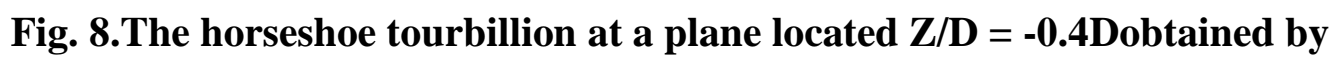
Realizable k- $\varepsilon$ 
The $\overline{u^{\prime} V^{\prime}} / U_{\text {cross }}^{2}$ fluctuations of the corresponding structures above and below the symmetryplane $y=0$ have evidently the same magnitude but differ in the sign, because $v^{\prime}$ changes the orientation at the symmetry-plane. The structures observed in these frames are possibly caused by the counter-rotating vortex pair. At lower heights below the top-plate, only the structures of the outer jet shear layer appear. At lower heights the jet is twisted by the crossflow to such an extent, that the recirculating inner shear-layer is underneath the measuring plane. Additionally, these outer structures above and below the symmetry-plane are moving outward each other in the figure, because the measuring plane cuts the jet at its "neck" and not parallel to the total cross-section area of the jet. As a further consequence, the separation between the structures visualized in the figures decreases in the flow direction (x-direction).

For the three components of the Reynolds stresses, at Lower highest the zones of high fluctuations are elongated as the jet is twisted by the cross flow. The physical quantities depicted in Figure 9 reflect the enhanced fluxes of a turbulent flow caused by the fluctuations of the turbulent flow. Therefore, they are of special concern for the development and validation of models describing turbulent flows.

\subsection{Degree Injection at Different Speed Ratio (VR)}

From Figure 10, a qualitative representation of the flow field is achieved including the extent of the jet deflection and the existence of impingement. In the initial region, the cross stream is deflected sideways in the initial region and accelerated around the edge of the jet and produces velocity maxima near side of the jet discharge. This velocity peak value is decreasing as VR increases. Increasing VR, the cross-flow is deflected very weakly by the jet.

Then jet stream gradually gains axial-direction momentum as it is convected downstream by the cross flow and the jet is aligned gradually with the cross stream. For VR $=2.776$ the region of the maximum axial velocity is expanded over a wide region and the flow is dominant by the cross-flow. In addition, the jet is deflected immediately at higher rate than the higher VR. As can be seen from Figure 10 for the specific VR the location where the jet trajectory intersects the measuring (x y) - plane and where the lower peak of the axial velocity are observed is shifted downstream at lower heights below the top plane because the jet is twisted by the momentum of the cross flow.

This behavior is significantly obvious for lower VR. The normalized axial velocity is used to represent the jet penetration. The jet penetration is inversely proportional to the local axial velocity. This figure shows that the penetration and the mixing of the jet with the cross-flow are significantly larger for higher VR. As shown in the Figure 10 the region of the minimum axial velocity is deflected downstream of the jet as VR deceases. For high value of VR the jet penetrates for longer distance through the cross flow as the negative axial velocity region is obvious for lower $x$ y planes. Such in the case of $\mathrm{VR}=5$ the low axial velocity region is appear for $\mathrm{x} y$ plane at $\mathrm{z} / \mathrm{D}=0.24$.

Note the wake regions in the cross-flow immediately upstream and downstream of the jet entrance. These regions are obstacles and they form blockage zone for the axial velocity. The zone of blockage of the jet to the cross-flow is generated by the recirculation zone which is known as horseshoe vortex. The wake region is induced by the backflow of the cross stream into the low-pressure region immediately downstream of the jet discharge. For example, at $\mathrm{VR}=2.776$ and $\mathrm{z} / \mathrm{D}=-0.24$ a recirculation zone appears at $\mathrm{x} / \mathrm{d}$ equals to 1 and 2 which is in consistent with the results of Figure 7. This wake region extends downstream but "lifts off" from the upper wall due to the strong inflow of the cross stream towards the symmetric plane, 


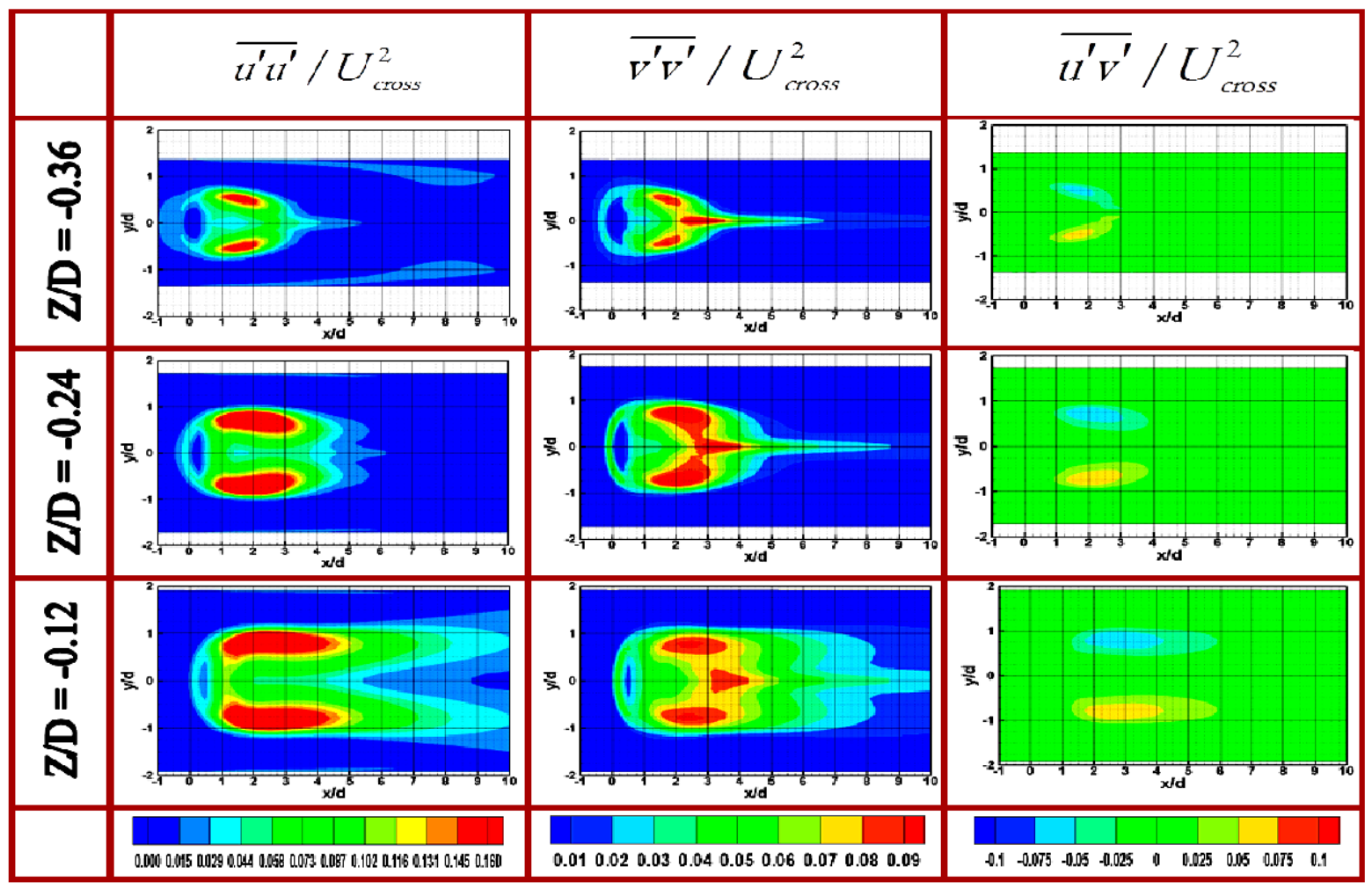

\section{Fig. 9. Two dimensional contours at a plane located at $0.5 \mathrm{~d}$ below the jet inlet obtained by RSM}

this effect is dominating for high value of velocity ratio such as VR $=4$ and 5 . However, these wake regions are formed for low VR only in the vicinity of the upper wall. This inward motion carries high-momentum fluid from the cross stream to the symmetric plane. Therefore, the axial component velocity profiles gradually smooth out downstream. The location of the strongest reverse flow is dependent on the rate of spreading of the cross-sectional area of the jet and, also, the location of maximum adverse pressure leeward of the jet.

At $\mathrm{VR}=2.776$, the jet is deflected downward near the jet discharge and more rapidly aligned with the cross stream. For flow dominated by the cross-flow velocity, the semi cylindrical recirculation zone behind the jet is greatly reduced as compared to higher VR especially at elevation of $\mathrm{z} / \mathrm{D}$ equal to 0 and disappears at $\mathrm{z} / \mathrm{D}=0.12$ where the entrainment rate of the cross-flow is high in the near field. With VR equals to 4 and 5, significant negative axial velocity motion continues farther downstream. For these higher VR a separation region is appeared just upstream the jet and represent the cross flow that is entrained by the jet. For $\mathrm{VR}=5$ this separation region is obvious for lower $\mathrm{x} y$ plane at $\mathrm{z} / \mathrm{D}=0.24$ and 0.36 , this effect is due to the recirculation caused by the jet impingement on the opposite wall. The upstream recirculation zone has disappearance at velocity ratio of 2.776 therefore the impingement does not occur.

It may be noticed that the flow mixture in the upstream zone has no immediate effect on the shear layer. Whereas, the low pressure region of the recirculation zone upstream of the jet has a strong effect on the jet trajectory. More particularly, on the boundary of the jet, the flow upstream created by the compressive stresses on the upper boundary of the jet, which triggers the formation of the CVP. The entrainment in the shear layer region of the jet represents the primary mechanism for deflecting the jet flow in the direction of the transverse flow. So, the entrainment is very important, not only to determine the mixing process but also to get an idea of the dynamic deviation of the jet. 
The interaction between the jet and the cross-flow produces a number of fluid dynamic structures. The counter-rotating vortex pair (CVP) originating at the jet exit is among the most important, since it is responsible of the cross-flow entrainment in the jet and appears to dominate convective mixing. Coherent structures like CVP can be identified by regions of high vorticity and low pressure.

The counter-rotating vortex pair (CVP), observed to dominate the jet cross-section, especially in the far field with evidence of its near field initiation. Cross flow shears the jet fluid along the lateral edges downstream to form a kidney shaped cross-section this shearing folds the downstream face over itself to form a vortex pair which dominates the far field flow. It should be noticed that the flow in this zone seems to be essentially conditioned by the pressure field at the jet exit. So, it is convenient to study the evolution of this vortex. The upright wake vortices which are formed within the wall boundary layer are shed beyond the JICF, allowing fluid to be drawn from the boundary layer into the jet itself. Mixing enhancement by the JICF is often associated with the development and sustenance of the CVP structures. Recalling from the turbulent and mean kinetic energy equation, the production term which is defined as the following:

$$
\tau_{i j} \frac{\partial U_{i}}{\partial x_{j}} \equiv\left|\rho \overline{u_{i} u_{j}}\right|\left|\frac{\partial U_{i}}{\partial x_{j}}\right|
$$

The maximum transfer energy from the mean flow to the turbulent flow occurs when the production term is maximized. Thus, the turbulent intensities which are an indication of the level of turbulence will be a maximum when the Reynolds stresses and mean velocity gradient are maximized.

In Figure 11, at different elevations the turbulent intensity maps obtained from the Realizable $\mathrm{k}-\varepsilon$ reveal the most characteristic flow feature of a JCF. At $V R=2.776, \mathrm{z} / \mathrm{D}=-0.24$ the counter-rotating vortex pair affect the turbulent intensity, because the jet penetrates perpendicular into the measuring plane. At lower heights below the top plate the jet is bent immediately by the cross flow towards the horizontal direction. As a consequence, this flow feature (CVP) cannot be easily recognized by measuring planes which are orientated parallel to the top plane. Therefore, this effect is not apparent in the lower planes.

As mentioned before, the highest degree of fluctuation is expected in the vicinity of the center of the jet trajectory. Therefore an intense mixing between the jet and the cross flow is also taking place. Increasing VR for the same height the region of the intense mixing between the jet and the cross is get closer to the jet inlet and the region of the high turbulent intensity is narrowed in the front boundary of the jet. For example, for VR $=5$ the high turbulent intensity is bounded in the narrow front head of the jet which is characterized by high velocity gradients until $\mathrm{z} / \mathrm{D}=0.12$. At low $\mathrm{VR}$ of 2.776 this region expanded and diffused downstream as the jet is significantly penetrated by the cross flow immediately at the jet exit. Also noting that, for higher VR, the narrower region of high turbulent intensity is bounded in the forehead of the jet especially at the high elevations and then expanded for lower elevations away from the jet inlet. This is a result of that; at higher VR the entrainment rate of the cross-flow through the jet is began in the far field. 


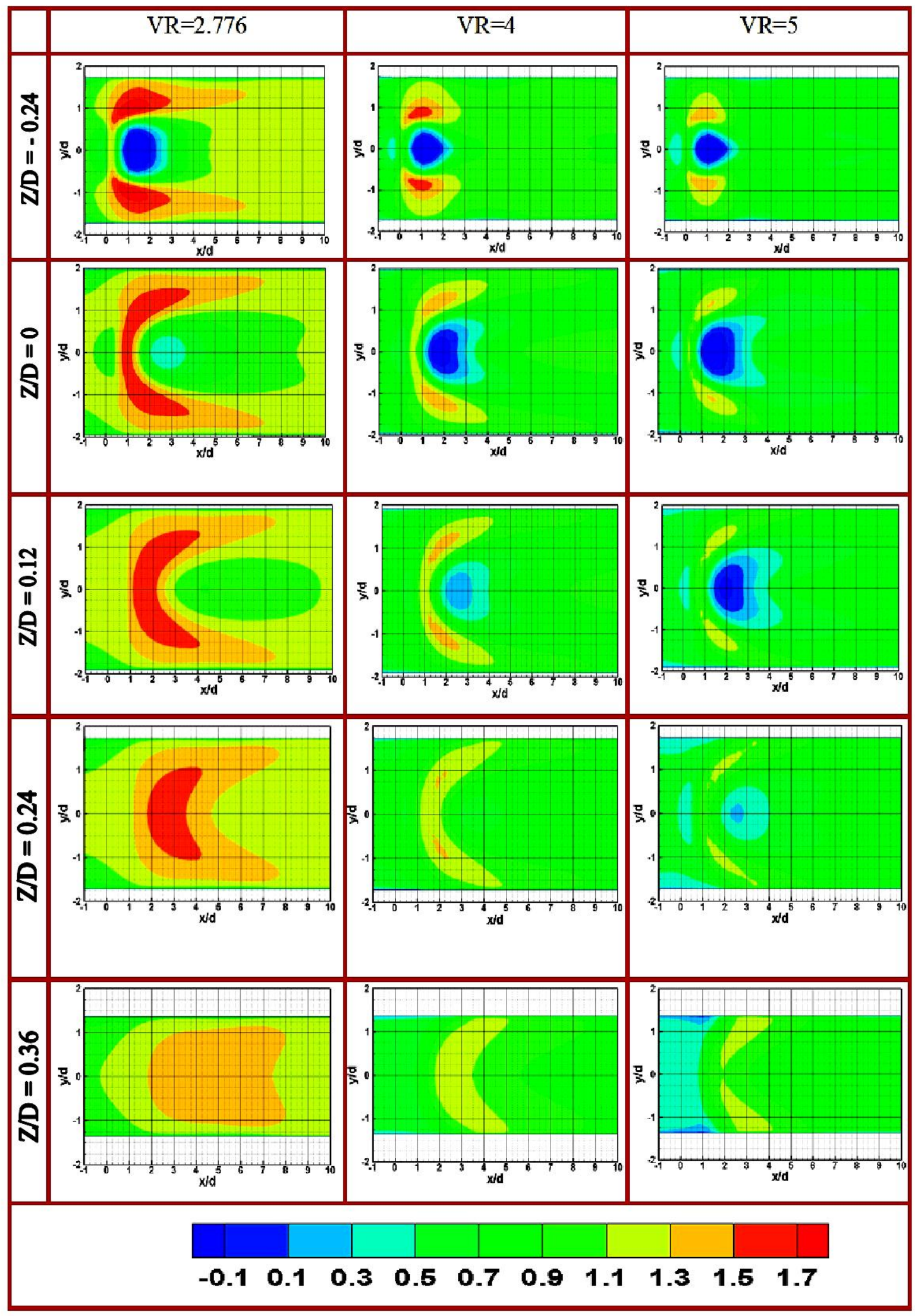

Fig. 10. Two dimensional contours of normalized axial velocity at different elevations below the jet inlet obtained by Realizable k- $\varepsilon$ 
Increasing VR with the effect of the CVP is appearing at further heights below the jet inlet and persists far downstream as the jet is able to penetrate for long distance through the cross flow. For example the "Kidney Shape" obvious for VR $=5$ for most elevations until z/D = 0.24 while for $\mathrm{VR}=4$ until elevation $\mathrm{z} / \mathrm{D}=0.12$. At $\mathrm{z} / \mathrm{D}=-0.24$ the effect of the CVP is more dominant for the VR $=2.776$ than the higher VR. This is a consequence of that; at lower VR the entrainment rate of the cross-flow through the jet is high in the near field.

For high VR the jet is penetrates for longer distance through the cross-flow which provides enough time for the mixing between the jet and the cross-flow. While for low VR the structures of the jet flow are eroded by the boundary layer before the interaction becomes possible. As can be seen from Figure $\mathbf{1 1}$ for the specific VR the location where the jet trajectory intersects the measuring (x y) plane and where the largest fluctuations are observed is shifted downstream at lower heights above the top plane because the jet is twisted by the momentum of the cross-flow. This behavior is significantly obvious for lower VR.

The results indicate that the diffusion characteristics of the jet stream strongly depend on the velocity ratio. For the lower velocity ratio, the jet is deflected rapidly by the influence of the cross-stream momentum. The jet stream is convected downstream and diffuses out in both the axial and transverse directions ( $\mathrm{x}$ and $\mathrm{y}$ directions, respectively). In the case of the higher velocity ratio, the jet stream diffuses more rapidly in the lateral direction (y direction). The kidney shaped cross section of the jet is clearly seen as the jet develops downstream. As shown in the Figure 11, decreasing the VR makes the outer shearing zone is drawn near to the wall at further axial distance. For example, at the center line $(\mathrm{z} / \mathrm{D}=0)$ the contacts occurs at $\mathrm{x} / \mathrm{d}$ equals to $2.5,3.75$, and 6.75 for VR equals to 5,4 and 2.776 respectively.

\section{Conclusion}

In this paper, the examinations of the influence of the jet flow into cross-flow coupling on the evolution of a transverse jet have been achieved. The simulate results have been validated by the experimental apparatus. The flow field has been simulated using a commercial code. The validation of the numerical simulation against experimental data to assess the ability of using the two-equation and RSM turbulent approaches to evaluate the structure of the jet flow into cross stream have been approved. In particular, the compression ware achieved at different positions of the jet axis. The comparison between the measured and simulated of customized form of Reynolds number $\left(\mathrm{Re}^{*}\right)$ results in the $\mathrm{x}$ and $\mathrm{z}$ directions show good agreement with the measurements. The velocity field contours are well represented by the three simulated turbulent models with a small advantage to the $\boldsymbol{k} \boldsymbol{-} \boldsymbol{\varepsilon}$ and SST models. The specific Reynolds stress component $\overline{u^{\prime} V^{\prime}}$ from the experimental and the numerical results show a lower degree of consistency.

The examinations of the JIC structure at different velocity ratio (VR) have been accomplished. The penetration and the mixing of the jet with the cross-flow are significantly larger for higher VR. Wake regions are formed in the cross-flow immediately upstream and downstream of the jet entrance. This wake region extends downstream but "lifts off" from the upper wall due to the strong inflow of the cross stream towards the symmetric plane, this effect is dominating for higher value of velocity ratio such as VR $=4$ and 5. However, these wake regions are formed for low VR only in the vicinity of the upper wall. For the higher VR a separation region appeares just upstream the jet and represent the cross-flow that is entrained by the jet. Finally, the jet stream is found to be convected downstream and diffuses out in both the axial and transverse directions ( $x$ and y directions, respectively). In the case of the higher velocity ratio, the jet stream diffuses more rapidly in the lateral direction (y direction). This effect is a result of that, as VR decreases the jet is curved by the transverse flow to be substantially horizontal relative to the wall, immediately downstream of the nozzle exit. 


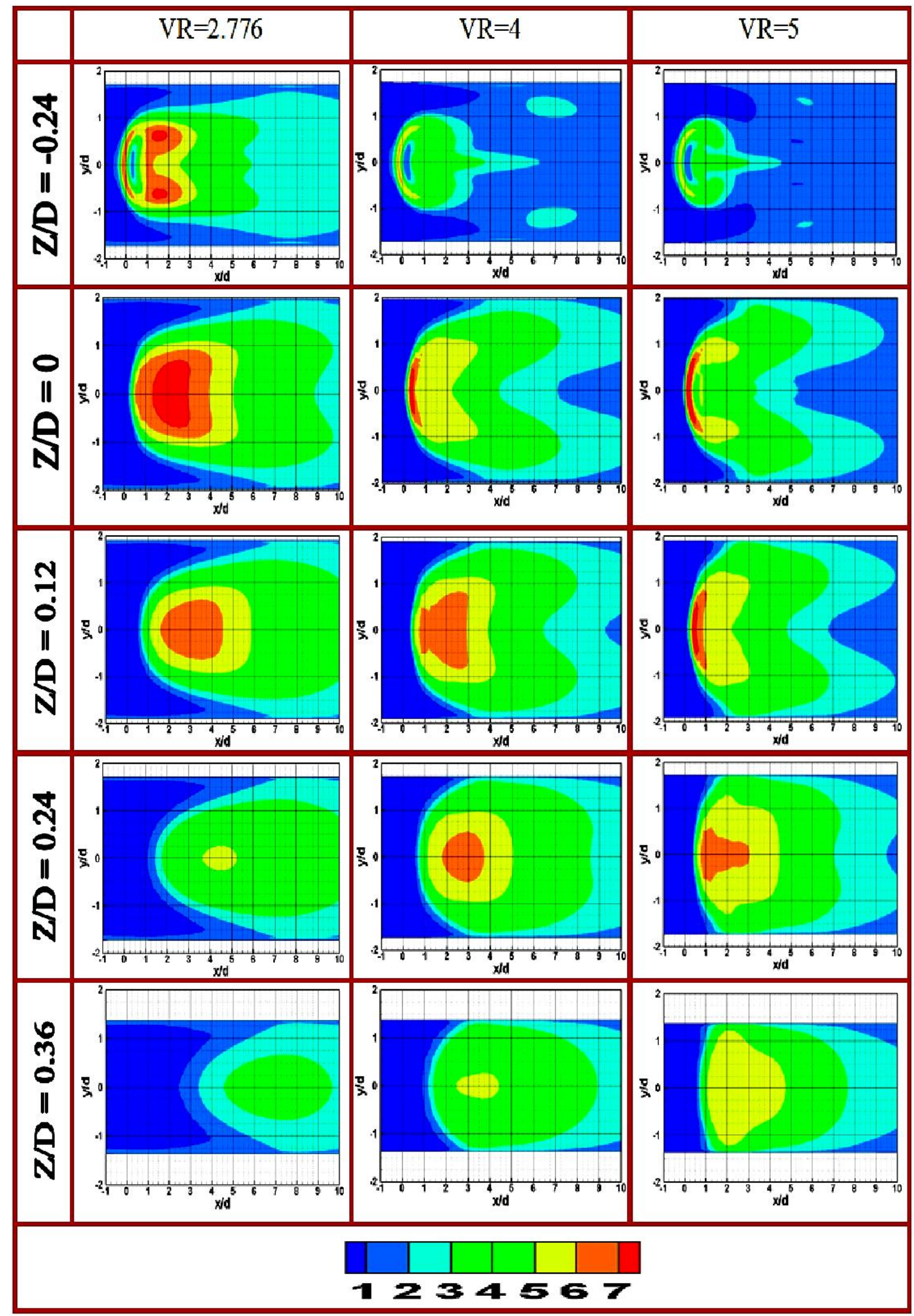

Fig. 11. Two dimensional contours of turbulent intensity at different elevations below the jet inlet obtained by Realizable k- $\varepsilon$ 


\section{References}

[1] A. O. Demuren, "Modeling turbulent jets in crossflow.," N.P. Cheremisinoff (Ed.), Encyclopedia of Fluid Mechanics, vol. 2, Gulf Publishing Company, Houston, TX,, vol. [Chapter 17], 1986.

[2] T. New, T. Lim, and S. Luo, "Elliptic jets in cross-flow," Journal of fluid mechanics, vol. 494, pp. 119-140, 2003.

[3] T. Fric and A. Roshko, "Vortical structure in the wake of a transverse jet," Journal of fluid mechanics, vol. 279, pp. 1-47, 1994.

[4] R. J. Margason, "Fifty years of jet in cross flow research," in In AGARD, Computational and Experimental Assessment of Jets in Cross Flow 41 p (SEE N94-28003 07-34), 1993.

[5] L. Su and M. Mungal, "Simultaneous measurements of scalar and velocity field evolution in turbulent crossflowing jets," Journal of fluid mechanics, vol. 513, pp. 1-45, 2004.

[6] C. Cárdenas, R. Suntz, and H. Bockhorn, "Experimental investigation of the mixingprocess in a jet-in-crossflow arrangement by simultaneous 2d-LIF and PIV," in Micro and Macro Mixing, ed: Springer, 2010, pp. 87-103.

[7] E. F. Hasselbrink and M. Mungal, "Transverse jets and jet flames. Part 2. Velocity and OH field imaging," Journal of fluid mechanics, vol. 443, pp. 27-68, 2001.

[8] C. Cárdenas, R. Suntz, J. A. Denev, and H. Bockhorn, "Two-dimensional estimation of Reynolds-fluxes and-stresses in a Jet-in-Crossflow arrangement by simultaneous 2D-LIF and PIV," Applied physics B, vol. 88, pp. 581-591, 2007.

[9] F. C. C. Galeazzo, G. Donnert, P. Habisreuther, N. Zarzalis, R. J. Valdes, and W. Krebs, "Measurement and simulation of turbulent mixing in a jet in crossflow," Journal of Engineering for Gas Turbines and Power, vol. 133, p. 061504, 2011.

[10] G. He, Y. Guo, and A. T. Hsu, "The effect of Schmidt number on turbulent scalar mixing in a jet-in-crossflow," International Journal of Heat and Mass Transfer, vol. 42, pp. 37273738, 1999.

[11] G. D. Catalano, K. Chang, and J. Mathis, "Investigation of turbulent jet impingement in a confined crossflow," AIAA journal, vol. 27, pp. 1530-1535, 1989.

[12] Y. Shi, M. Ray, and A. Mujumdar, "Numerical study on the effect of cross-flow on turbulent flow and heat transfer characteristics under normal and oblique semi-confined impinging slot jets," Drying technology, vol. 21, pp. 1923-1939, 2003.

[13] "ANSYS Fluent User's Guide, Release 16.0, 2015."

[14] D. C. Wilcox, Turbulence modeling for CFD vol. 2: DCW industries La Canada, CA, 1998.

[15] T.-H. Shih, W. W. Liou, A. Shabbir, Z. Yang, and J. Zhu, "A new k- $\epsilon$ eddy viscosity model for high reynolds number turbulent flows," Computers \& Fluids, vol. 24, pp. 227238, 1995.

[16] F. R. Menter, "Two-equation eddy-viscosity turbulence models for engineering applications," AIAA journal, vol. 32, pp. 1598-1605, 1994.

[17] B. Launder, G. J. Reece, and W. Rodi, "Progress in the development of a Reynolds-stress turbulence closure," Journal of fluid mechanics, vol. 68, pp. 537-566, 1975.

[18] B. E. Launder, "Second-moment closure: present... and future?," International Journal of Heat and Fluid Flow, vol. 10, pp. 282-300, 1989.

[19] F.-S. Lien and M. Leschziner, "Assessment of turbulence-transport models including nonlinear RNG eddy-viscosity formulation and second-moment closure for flow over a backward-facing step," Computers \& Fluids, vol. 23, pp. 983-1004, 1994.

[20] C. G. Speziale, S. Sarkar, and T. B. Gatski, "Modelling the pressure-strain correlation of turbulence: an invariant dynamical systems approach," Journal of fluid mechanics, vol. 227, pp. 245-272, 1991.

[21] A. R. Karagozian, "Background on and Applications of Jets in Crossflow," in Manipulation and Control of Jets in Crossflow, ed: Springer, 2003, pp. 3-13. 